\title{
Bioabsorbable Implant for Treatment of Nasal Valve Collapse with or without Concomitant Procedures
}

\author{
Douglas M. Sidle, MD ${ }^{1}$ Pablo Stolovitzky, MD² Ellen M. O’Malley, MS ${ }^{3}$ Randall A. Ow, MD \\ Nathan E. Nachlas, MD ${ }^{5}$ Stacey Silvers, MD
}

${ }^{1}$ Department of Otolaryngology-Head and Neck Surgery, Northwestern University Feinberg School of Medicine, Chicago, Illinois

2 Department of Otolaryngology, Emory University School of Medicine, Atlanta, Georgia

${ }^{3}$ ENT Clinical Affairs, Stryker Corp, Plymouth, Minnesota

${ }^{4}$ Sacramento Ear Nose and Throat Medical and Surgical Group, Roseville, California

${ }^{5}$ Ear, Nose, Throat, and Allergy Associates of Florida, Boca Raton, Florida

6 Madison ENT and Facial Plastic Surgery, New York, New York

\begin{abstract}
Address for correspondence Douglas M. Sidle, MD, Department of Otolaryngology-Head and Neck Surgery, Northwestern University Feinberg School of Medicine, 676 N. St Clair Street, Suite 1325, Chicago, IL 60611 (e-mail: d.sidle@nm.org).
\end{abstract}

Facial Plast Surg 2021;37:673-680.

\author{
Abstract \\ Keywords \\ - nasal valve collapse \\ - nasal airway \\ obstruction \\ - bioabsorbable \\ implant \\ - lateral wall \\ insufficiency \\ - nasal valve repair
}

The aim of the study is to report outcomes after treatment of nasal valve collapse with a bioabsorbable nasal implant. It involves two prospective, multicenter, post-market studies evaluating long-term effectiveness of the LATERA implant for severe to extreme nasal obstruction. Participants underwent implant alone or with concomitant inferior turbinate reduction (ITR) and/or septoplasty. Outcome measures included the change from baseline Nasal Obstruction Symptom Evaluation (NOSE) scores, NOSE responder rates, visual analog scale (VAS) scores, and adverse events. A total cohort of 277 participants (109 implants only, 67 implants + ITR, 101 implants + septoplasty + ITR) enrolled at 19 U.S. centers was available for analysis with 177 participants (69 implants only, 39 implants + ITR, 69 implants + septoplasty + ITR) available at 2 years. The mean changes from baseline in NOSE scores and VAS scores were statistically significant $(p<0.001)$ at all follow-up periods. The baseline NOSE score of $77.8 \pm 13.6$ was improved to $24.2 \pm 23.6$ at 24 months. Greater than $90 \%$ of participants were NOSE responders across all follow-up periods, $6.1 \%$ withdrew for lack of treatment effect. The baseline VAS score of $66.7 \pm 18.8$ was improved to $21.1 \pm 23.9$ at 24 months. There were no serious adverse events related to the device or implant procedure. Implant retrieval rate was $4.0 \%$ (22/543 implants). Nonserious adverse events were mild to moderate in severity, typically occurred within 6 months of implant, and resolved or were stable. Significant reductions in NOSE and VAS scores and high responder rates from our large population of patients with nasal obstruction who had nasal valve implants confirm sustained effectiveness at 24 months after treatment. The studies are registered on www.clinicaltrials.gov (NCT02952313 and NCT02964312). published online April 14, 2021
Issue Theme Facial Plastic Surgery Original Research; Guest Editors: Anthony P. Sclafani, MD, MBA, FACS, and Alwyn D'Souza, MBBS, FRCS Eng, FRCS (ORL-HNS)

DOI https://doi.org/ 10.1055/s-0041-1726464. ISSN $0736-6825$. (c) 2021. The Author(s).

This is an open access article published by Thieme under the terms of the Creative Commons Attribution-NonDerivative-NonCommercial-License, permitting copying and reproduction so long as the original work is given appropriate credit. Contents may not be used for commercial purposes, or adapted, remixed, transformed or built upon. (https://creativecommons.org/ licenses/by-nc-nd/4.0/)

Thieme Medical Publishers, Inc., 333 Seventh Avenue, 18th Floor, New York, NY 10001, USA 
Nasal obstruction is a common problem that leads to significant impairment of quality of life for patients. ${ }^{1}$ The most prominent anatomical causes of nasal obstruction are septal deviation, turbinate hypertrophy, and nasal valve collapse (NVC). A recent survey of 50 U.S. physicians reported that, in patients with severe to extreme Nasal Obstructive Symptoms Evaluation (NOSE) scores, the prevalence of these anatomical conditions was $80 \%$ for septal deviation, $77 \%$ for turbinate hypertrophy, and $73 \%$ for NVC. ${ }^{2}$ Up to $82 \%$ of patients with severe to extreme nasal obstruction who have had previous septoplasty and/or inferior turbinate reduction (ITR) have $\mathrm{NVC}^{2}$ suggesting it is frequently overlooked as a causative factor of nasal obstruction. Chambers et al reported significant improvement after nasal valve repair in patients who had failed to respond to septoplasty. ${ }^{3}$

In 2016, the U.S. Food and Drug Administration cleared a bioabsorbable implant for supporting upper and lower lateral nasal cartilages. ${ }^{4}$ The procedure is minimally invasive and can be performed under local anesthesia. ${ }^{5}$ Several studies have reported the safety and efficacy of the implant with follow-up of up to 12 months. ${ }^{6-8}$ The first-in-human study, conducted in Germany, reported 24-month follow-up for population of 30 patients. ${ }^{9}$ For this paper, we report outcomes through 24 months on a large combined population from two related multicenter U.S. studies. Additionally, we evaluated outcomes in subgroups of participants who underwent implant placement only compared with participants who underwent implant placement with concomitant procedures (i.e., ITR, septoplasty).

\section{Materials and Methods}

\section{Study Design and Population}

Two prospective, multicenter, nonrandomized, interventional, post-market studies were designed to evaluate the LATERA absorbable nasal implant (Stryker ENT, Plymouth MN 55447) as a treatment for patients with severe to extreme nasal obstruction. The methods and early outcomes of these studies have been previously reported. ${ }^{7,8}$ Briefly, the two studies used similar criteria to enroll participants with nasal obstruction that was predominantly due to NVC. One study enrolled participants for implant treatment in the operating room with the option for concomitant ITR and/or septoplasty; the other study enrolled participants for in-office implant treatment with the option for concomitant ITR only. The need for concomitant procedures was at the discretion of the investigator based on the individual participant's clinical needs and preferences. Other assessments, procedures, and end points were identical between the studies. A total of 19 centers participated in the two studies with participants enrolled between September 2016 and August 2017. The Schulman IRB (now Advarra IRB) or a local IRB approved the study for all centers and written informed consent was obtained from all participants. The studies are registered on www.clinicaltrials.gov (NCT02952313 and NCT02964312).

Participants were adults ( $\geq 18$ years) with severe to extreme nasal obstruction (NOSE score $\geq 55$ ) and a positive modified Cottle maneuver who were seeking treatment after failing to benefit from or intolerant to appropriate maximal medical management (e.g., 4-week course of nasal steroids; antihistamines; oral decongestants; nasal strips, stents, or cones). Participants were also evaluated to ensure they had appropriate nasal and facial anatomy to receive the implant.

Participants were excluded from the study if they required concurrent functional endoscopic sinus surgery or sinuplasty; had rhinoplasty within the past 12 months or were planning to have a rhinoplasty procedure or use external dilators at any time during the study duration; had septoplasty and/or ITR within the past 6 months; had inappropriate fixation for fixed nasal valve obstruction or collapse due to significant scarring; had a permanent implant or dilator in the nasal area; was a chronic systemic steroid or recreational intranasal drug user; or had inflammatory or infectious skin or nasal conditions. Participants were also excluded if they had cancerous/precancerous lesions or had undergone radiation or chemotherapy in the treatment area; had polyps or other pathology that would contribute to airway obstruction; had a history of a significant bleeding disorder(s); had a known or suspected allergy to polylactide (PLA) or other absorbable materials; had a significant systemic disease that could predispose to poor wound healing; or required nasal oxygen or continuous positive airway pressure. Pregnant or lactating females were also excluded.

All participants underwent unilateral or bilateral placement of the bioabsorbable implant. Only one implant was allowed per side. The implant is FDA-cleared with the indication for supporting the upper and lower lateral nasal cartilage. The implant was used according to the cleared indication for use.

\section{Assessments}

We report the following outcomes from all follow-up visits (at months 1, 3, 6, 12,18, and 24) for the combined study population: change from baseline in NOSE scores, NOSE responder rates, change in nasal breathing visual analog scale (VAS) scores, and adverse events. Participants were free to discontinue the study at any time for any reason, including failure to respond to the treatment.

The NOSE tool is a validated patient-reported outcome with a total score ranging from 0 to $100 .^{10}$ A severity classification scheme for the total NOSE scores has been developed by Lipan and Most. ${ }^{11}$ Scores of 5 to 25 are considered mild, 30 to 50 are moderate, 55 to 75 are severe, and 80 to 100 are extreme. We defined a NOSE responder as a participant who has improvement of 1 or more NOSE classes or $20 \%$ or more reduction from baseline in NOSE score.

At baseline and follow-up visits, participants completed a nasal breathing VAS, with $0 \mathrm{~mm}$ representing no symptoms and $100 \mathrm{~mm}$ representing severe symptoms.

All serious and nonserious device-/procedure-related adverse events were reported from treatment through the 24month follow-up. All events reported as possibly, probably, or definitely related to the device, procedure, or both were defined as related events. All adverse events were adjudicated by a medical monitor. 


\section{Statistical Analysis}

Baseline characteristics, including demographic characteristics, surgical history, and patient-reported outcomes are summarized using descriptive statistics. Discrete variables are expressed as rates and proportions; continuous variables are reported as means and standard deviations. Each participant serves as their own control, and changes from baseline are calculated using mixed models for repeated measures and reported as least-square means with $95 \%$ confidence intervals (CIs). $P$ values are based on two-sided paired $t$-tests with 0.05 indicating statistical significance.

Subgroup analysis of the primary end point was performed based on the presence or absence of concomitant sinonasal procedures.

To address the impact of missing data on the 24-month outcomes, a worst-case sensitivity analysis was conducted where all participants with a missing 24-month visit for any reason were assigned no change from baseline for the NOSE and VAS scores. The mean change from baseline and $95 \% \mathrm{CI}$ were calculated.

Statistical analyses were performed by an independent statistician using SAS version 9.4.

\section{Results}

\section{Participants}

A total of 279 participants were enrolled at 19 U.S. centers. Two participants withdrew before the 1-month follow-up due to implant retrievals and are, therefore, not included in the efficacy analyses resulting in a total of 277 analyzed participants. Demographics and other baseline data are presented in - Table 1.

In the combined population, 109 participants (39.4\%) received the implant only, $67(24.2 \%)$ received the implant with a concomitant ITR, and 101 (36.5\%) received the implant and septoplasty with or without ITR. Mechanical reduction
(50\%) and radiofrequency ablation (44\%) were the most common ITR methods used. Bilateral implants were placed in most participants (95\%).

Participant follow-up throughout the study is shown in - Table 2. Follow-up through the initial protocol followup was very good with $83.1 \%$ (232/279) of participants completing the 12-month visit. A protocol amendment that required reconsent was incorporated to continue follow-up at 18 and 24 months. A total of 191 participants consented to the amended protocol with 177 participants completing the study through 24 months. One-hundred participants discontinued early: 44 (15.9\% of the total enrollment) were lost to follow-up, $16(5.8 \%)$ withdrew for reasons unrelated to NAO, 17 (6.1\%) withdrew due to lack of treatment response, $21(7.6 \%)$ did not consent for the longterm extension protocol, and two $(0.7 \%)$ died from causes unrelated to the study.

\section{Patient-Reported Outcomes}

In concordance with enrollment criteria, all participants had severe to extreme baseline NOSE scores with a mean score of $77.8 \pm 13.6$. After treatment, the mean NOSE scores ranged from $33.7 \pm 23.0$ at 1 month (mean change -43.9 ) to $24.2 \pm 23.6$ at 24 months (mean change -53.6 ). The change from baseline was statistically significant $(p<0.001)$ at all follow-up periods. Inclusion of the two patients who withdrew prior to 1 month and imputing 0 for their change did not change the results appreciably ( 1 month, mean change $-43.8 ; 24$ months, mean change -53.3 ). The change in mean NOSE scores for all participants and for each treatment group is presented in - Table 3 and -Fig. 1 .

The worst-case sensitivity analysis, assuming all 102 participants without a 24-month visit had no change from baseline demonstrated a persistent significant reduction in the NOSE score (mean change $-34.2 ; 95 \% \mathrm{CI},-38.1,-30.2$ ) and VAS score (mean change $-28.7 ; 95 \% \mathrm{CI},-32.3,-25.2$ ).

Table 1 Demographics and baseline characteristics

\begin{tabular}{|l|l|l|l|l|}
\hline Characteristic & $\begin{array}{l}\text { Implant only } \\
\mathbf{N}=\mathbf{1 0 9}\end{array}$ & $\begin{array}{l}\text { Implant + ITR } \\
\mathbf{N}=\mathbf{6 7}\end{array}$ & $\begin{array}{l}\text { Implant }+ \text { septoplasty }+ \text { ITR }^{\mathrm{a}} \\
\mathbf{N}=\mathbf{1 0 1}\end{array}$ & $\begin{array}{l}\text { All participants } \\
\mathbf{N}=\mathbf{2 7 7}\end{array}$ \\
\hline Age (years) & $51.0 \pm 14.3$ & $49.2 \pm 15.6$ & $45.0 \pm 13.7$ & $48.4 \pm 14.6$ \\
\hline Sex (male) & $49.5 \%(54 / 109)$ & $50.7 \%(34 / 67)$ & $58.4 \%(5 / 101)$ & $53.1 \%(147 / 277)$ \\
\hline Race (White) & $88.1 \%(96 / 109)$ & $80.6 \%(54 / 67)$ & $86.1 \%(87 / 101)$ & $85.6 \%(237 / 277)$ \\
\hline Ethnicity (Hispanic) & $8.3 \%(9 / 109)$ & $10.4 \%(7 / 67)$ & $9.0 \%(9 / 101)$ & $9.1 \%(25 / 277)$ \\
\hline NOSE & $77.8 \pm 13.7$ & $77.2 \pm 13.2$ & $78.3 \pm 13.8$ & $77.8 \pm 13.6$ \\
\hline Nasal symptoms VAS & $69.8 \pm 18.1$ & $65.0 \pm 17.5$ & $64.8 \pm 20.4$ & $66.7 \pm 18.8$ \\
\hline Previous sinonasal surgery & $72.5 \%(79 / 109)$ & $40.3 \%(27 / 67)$ & $15.8 \%(16 / 101)$ & $44.0 \%(122 / 277)$ \\
\hline Septoplasty & $72.4 \%(55 / 79)$ & $56.0 \%(14 / 27)$ & $57.1 \%(8 / 16)$ & $67.0 \%(77 / 122)$ \\
\hline Turbinate reduction & $69.7 \%(53 / 79)$ & $60.0 \%(15 / 27)$ & $42.9 \%(6 / 16)$ & $64.3 \%(74 / 122)$ \\
\hline Rhinoplasty & $14.5 \%(11 / 79)$ & $12.0 \%(3 / 27)$ & $64.3 \%(9 / 16)$ & $20.0 \%(23 / 122)$ \\
\hline Endoscopic sinus surgery & $35.5 \%(27 / 79)$ & $40.0 \%(10 / 27)$ & $35.7 \%(5 / 16)$ & $36.5 \%(42 / 277)$ \\
\hline
\end{tabular}

Abbreviations: ITR, inferior turbinate reduction; NOSE, Nasal Obstruction Symptom Evaluation; VAS, visual analog scale.

Note: Results are presented as mean \pm SD or $\%(n / N)$.

${ }^{a}$ Four participants who had implant plus septoplasty without concomitant ITR are included. 
Table 2 Participant flow

\begin{tabular}{|c|c|c|c|c|}
\hline Follow-up period & Number and reason for exit before visit & Number active & $\begin{array}{l}\text { Number of } \\
\text { missed visits }\end{array}$ & $\begin{array}{l}\text { Number } \\
\text { analyzable }\end{array}$ \\
\hline Treatment & 0 & 279 & 0 & 279 \\
\hline $1 \mathrm{mo}$ & 2 implants retrieved, no follow-up & 277 & 1 & 276 \\
\hline $3 \mathrm{mo}$ & $\begin{array}{l}3 \text { LTFU } \\
1 \text { WD non-NAO } \\
1 \text { WD NAO }\end{array}$ & 272 & 5 & 267 \\
\hline $6 \mathrm{mo}$ & $\begin{array}{l}5 \text { LTFU } \\
2 \text { WD non-NAO } \\
4 \text { WD NAO }\end{array}$ & 261 & 1 & 260 \\
\hline $12 \mathrm{mo}$ & $\begin{array}{l}16 \text { LTFU } \\
7 \text { WD non-NAO } \\
4 \text { WD NAO } \\
1 \text { unrelated death }\end{array}$ & 233 & 1 & 232 \\
\hline $18 \mathrm{mo}$ & $\begin{array}{l}7 \text { LTFU } \\
6 \text { WD non-NAO } \\
7 \text { WD NAO } \\
1 \text { unrelated death } \\
21 \text { did not reconsent to LTFU }\end{array}$ & 191 & 6 & 185 \\
\hline $24 \mathrm{mo}$ & $\begin{array}{l}13 \text { LTFU } \\
1 \text { WD NAO }\end{array}$ & 177 & 0 & 177 \\
\hline
\end{tabular}

Abbreviations: LTFU, lost to follow-up; WD NAO, withdrew due to additional nasal airway obstruction surgery required; WD non-NAO, withdrew for reasons unrelated to nasal airway obstruction.

${ }^{\text {a}}$ The 18- and 24-mo visits were part of an extension protocol which required additional consent.

In the full population, the percentage of participants who met the definition of NOSE responders was $>90 \%$ across all follow-up periods ( - Table 4). Participants treated with the nasal implant alone had response rates similar to those participants who underwent the implant with concomitant ITR (88.3-94.5\% vs. 88.1-94.9\%). Participants who required septoplasty in addition to the nasal implant, with or without ITR, had responder rates ranging from 93.0 to $95.8 \%$.

The baseline mean nasal breathing VAS score was $66.7 \pm 18.8$ for the full population. Post-treatment mean scores ranged from $29.8 \pm 24.2$ at 1 month (mean change -36.6 ) to $21.1 \pm 23.9$ at 24 months (mean change -45.3 ). VAS scores for all participants are presented in -Table 5 .

\section{Adverse Events}

There were no serious adverse events related to the device or implant procedure. A total of 54 nonserious device-/procedure-related events were reported in 45 participants; all were mild or moderate in severity and resolved without clinical sequelae or were ongoing but stable at the end of the study. By procedure group, the AE rates were $21.1 \%$ (23/109) for the implant only group, $17.9 \%$ (12/67) for the implant + ITR group, and 9.9\% (10/101) for the implant + ITR + septoplasty group. The device-/procedure-related adverse events are listed in $\mathbf{- T a b l e} \mathbf{6}$. The most common event reported was implant retrieval/extrusion with an implant retrieval rate of 4.0\% (22/543) per implant or 7.9\% (22/277) per participant. All but one of the implant extrusions/retrievals were through the nostril and none of them required general anesthesia for removal. The most common cause of retrievals was partial exposure of the device at the insertion point. Only one partial exposure was through the skin at a site remote from the insertion site. Ten of the extrusion/retrievals were reported by the participant to have occurred at home and 12 were observed directly by the investigator in the clinic. There was only one report of undesired cosmetic changes (bumps on the nose) after the 12-month period. Related adverse events are rare $(1.5 \%, 4 / 264)$ after the initial 6 months post implant.

\section{Discussion}

We report the long-term outcomes of a large population of patients with nasal obstruction who underwent placement of an absorbable nasal valve implant with or without concomitant nasal procedures. Our findings validate earlier studies of the implant in a smaller population of patients and confirm the long-term effectiveness of the implant. ${ }^{6,9}$

Rhee et al reported a meta-analysis of 31 articles reporting pre and/or postsurgical (e.g., ITR, septoplasty) NOSE and VAS scores. ${ }^{12}$ They reported a presurgical weighted mean NOSE score of $65 \pm 22$. The postsurgical average was $23 \pm 20$, resulting in a mean change from baseline 42 points. Their weighted presurgical mean VAS was $6.7 \pm 2.3$ (on a scale of $0-10$ ). The postsurgical mean was $2.1 \pm 2.2$, resulting in a mean change from baseline of 4.6. These findings are consistent with our study findings of mean NOSE reductions ranging from 43.9 to 53.6 and VAS reductions of 36.6 to 45.3 ( $0-100$ scale).

Samra et al reported on a variety of surgical techniques to manage NVC. ${ }^{5}$ Spreader grafts, alar batten grafts, and various open septorhinoplasty techniques tailored to specific deficiencies in the lateral nasal wall are described. These surgical 
Table 3 Change in mean NOSE score by treatment group

\begin{tabular}{|c|c|c|c|c|c|}
\hline Follow-up period & $N$ & Baseline NOSE score & Follow-up NOSE score & $\begin{array}{l}\text { LS mean change in } \\
\text { NOSE score }(95 \% \mathrm{Cl})\end{array}$ & p-Value \\
\hline \multicolumn{6}{|l|}{ Implant only } \\
\hline $1 \mathrm{mo}$ & 109 & $77.8 \pm 13.7$ & $37.0 \pm 21.9$ & $-40.5(-44.8 ;-36.2)$ & $<0.001$ \\
\hline $3 \mathrm{mo}$ & 106 & $77.6 \pm 13.7$ & $33.0 \pm 22.7$ & $-44.5(-48.8 ;-40.1)$ & $<0.001$ \\
\hline $6 \mathrm{mo}$ & 100 & $77.3 \pm 13.7$ & $32.1 \pm 22.7$ & $-45.3(-49.8 ;-40.8)$ & $<0.001$ \\
\hline $12 \mathrm{mo}$ & 94 & $76.4 \pm 13.6$ & $32.6 \pm 24.1$ & $-44.7(-49.4 ;-40.1)$ & $<0.001$ \\
\hline $18 \mathrm{mo}$ & 73 & $77.9 \pm 13.2$ & $30.1 \pm 22.9$ & $-47.4(-52.7 ;-42.1)$ & $<0.001$ \\
\hline $24 \mathrm{mo}$ & 69 & $77.8 \pm 13.4$ & $30.4 \pm 24.6$ & $-47.1(-52.6 ;-41.7)$ & $<0.001$ \\
\hline \multicolumn{6}{|l|}{ Implant + ITR } \\
\hline $1 \mathrm{mo}$ & 67 & $77.2 \pm 13.2$ & $35.1 \pm 24.1$ & $-42.0(-47.7 ;-36.2)$ & $<0.001$ \\
\hline $3 \mathrm{mo}$ & 62 & $77.3 \pm 12.9$ & $32.5 \pm 24.7$ & $-44.6(-50.6 ;-38.6)$ & $<0.001$ \\
\hline $6 \mathrm{mo}$ & 63 & $77.3 \pm 13.0$ & $33.2 \pm 28.3$ & $-44.0(-49.9 ;-38.0)$ & $<0.001$ \\
\hline $12 \mathrm{mo}$ & 54 & $77.2 \pm 12.9$ & $27.2 \pm 24.1$ & $-49.9(-56.3 ;-43.5)$ & $<0.001$ \\
\hline $18 \mathrm{mo}$ & 45 & $75.7 \pm 12.6$ & $31.2 \pm 25.1$ & $-45.0(-52.0 ;-37.9)$ & $<0.001$ \\
\hline $24 \mathrm{mo}$ & 39 & $76.5 \pm 12.2$ & $27.6 \pm 23.1$ & $-49.1(-56.7 ;-41.6)$ & $<0.001$ \\
\hline \multicolumn{6}{|c|}{ Implant + Septoplasty + ITR ${ }^{a}$} \\
\hline $1 \mathrm{mo}$ & 100 & $78.2 \pm 13.8$ & $29.2 \pm 22.9$ & $-49.0(-53.2 ;-44.9)$ & $<0.001$ \\
\hline $3 \mathrm{mo}$ & 99 & $78.1 \pm 13.8$ & $19.2 \pm 20.8$ & $-59.0(-63.2 ;-54.9)$ & $<0.001$ \\
\hline $6 \mathrm{mo}$ & 95 & $78.2 \pm 13.9$ & $18.8 \pm 19.5$ & $-59.4(-63.7 ;-55.2)$ & $<0.001$ \\
\hline $12 \mathrm{mo}$ & 84 & $77.4 \pm 14.0$ & $17.9 \pm 21.1$ & $-60.2(-64.8 ;-55.7)$ & $<0.001$ \\
\hline $18 \mathrm{mo}$ & 67 & $78.7 \pm 13.6$ & $16.3 \pm 21.9$ & $-61.9(-67.0 ;-56.9)$ & $<0.001$ \\
\hline $24 \mathrm{mo}$ & 69 & $79.1 \pm 13.3$ & $16.0 \pm 20.7$ & $-62.3(-67.3 ;-57.3)$ & $<0.001$ \\
\hline \multicolumn{6}{|l|}{ All participants } \\
\hline $1 \mathrm{mo}$ & 276 & $77.8 \pm 13.6$ & $33.7 \pm 23.0$ & $-43.9(-46.7,-41.2)$ & $<0.001$ \\
\hline $3 \mathrm{mo}$ & 267 & $77.7 \pm 13.5$ & $27.8 \pm 23.4$ & $-49.9(-52.7,-47.1)$ & $<0.001$ \\
\hline $6 \mathrm{mo}$ & 258 & $77.6 \pm 13.6$ & $27.5 \pm 24.0$ & $-50.2(-53.0,-47.3)$ & $<0.001$ \\
\hline $12 \mathrm{mo}$ & 232 & $77.0 \pm 13.5$ & $26.0 \pm 23.9$ & $-51.5(-54.5,-48.4)$ & $<0.001$ \\
\hline $18 \mathrm{mo}$ & 185 & $77.6 \pm 13.2$ & $25.4 \pm 24.0$ & $-52.2(-55.6,-48.8)$ & $<0.001$ \\
\hline $24 \mathrm{mo}$ & 177 & $78.0 \pm 13.1$ & $24.2 \pm 23.6$ & $-53.6(-57.0,-50.1)$ & $<0.001$ \\
\hline
\end{tabular}

Abbreviations: ITR, inferior turbinate reduction; LS, least squares; NOSE, Nasal Obstruction Symptom Evaluation; SD, standard deviation. Note: Results are presented as mean \pm SD.

${ }^{\text {a } F o u r ~ p a r t i c i p a n t s ~ w h o ~ h a d ~ i m p l a n t ~ p l u s ~ s e p t o p l a s t y ~ w i t h o u t ~ c o n c o m i t a n t ~ I T R ~ a r e ~ i n c l u d e d . ~}$

techniques can be challenging, and results are highly related to experience. Complications can be difficult to correct. The availability of a minimally invasive implant that can be placed in the office-setting offers an attractive alternative treatment option that general otolaryngologists and subspecialists alike can offer to their patients with NVC.

There is a risk of device exposure or extrusion through the insertion site shortly after the procedure, which should be monitored. This may be partially due to the learning curve associated with the implant procedure with extrusion/retrieval occurring if the implant is left too close to the insertion site. The manufacturer's instructions for use recommend counseling patients to avoid post-procedure manipulation of the nose during the acute healing period to help prevent extrusions (e.g., week 1: do not pinch or blow nose; weeks 1-2: avoid strenuous activity; weeks 1-4: do not place objects inside of nose). When partial exposure occurs, the device can be trimmed or removed with a forceps without difficulty.

An advantage of the combined population of these two studies is the ability to evaluate outcomes based on the concomitant procedures performed. Previous studies have demonstrated that failure to consider the nasal valve contribution to nasal obstruction is a contributing factor to treatment failure after septoplasty. ${ }^{2,3}$ Participants were permitted to have ITRs and/or septoplasties based on each person's individual needs. Although this creates an inherent selection bias for the subgroup analysis, it is also 


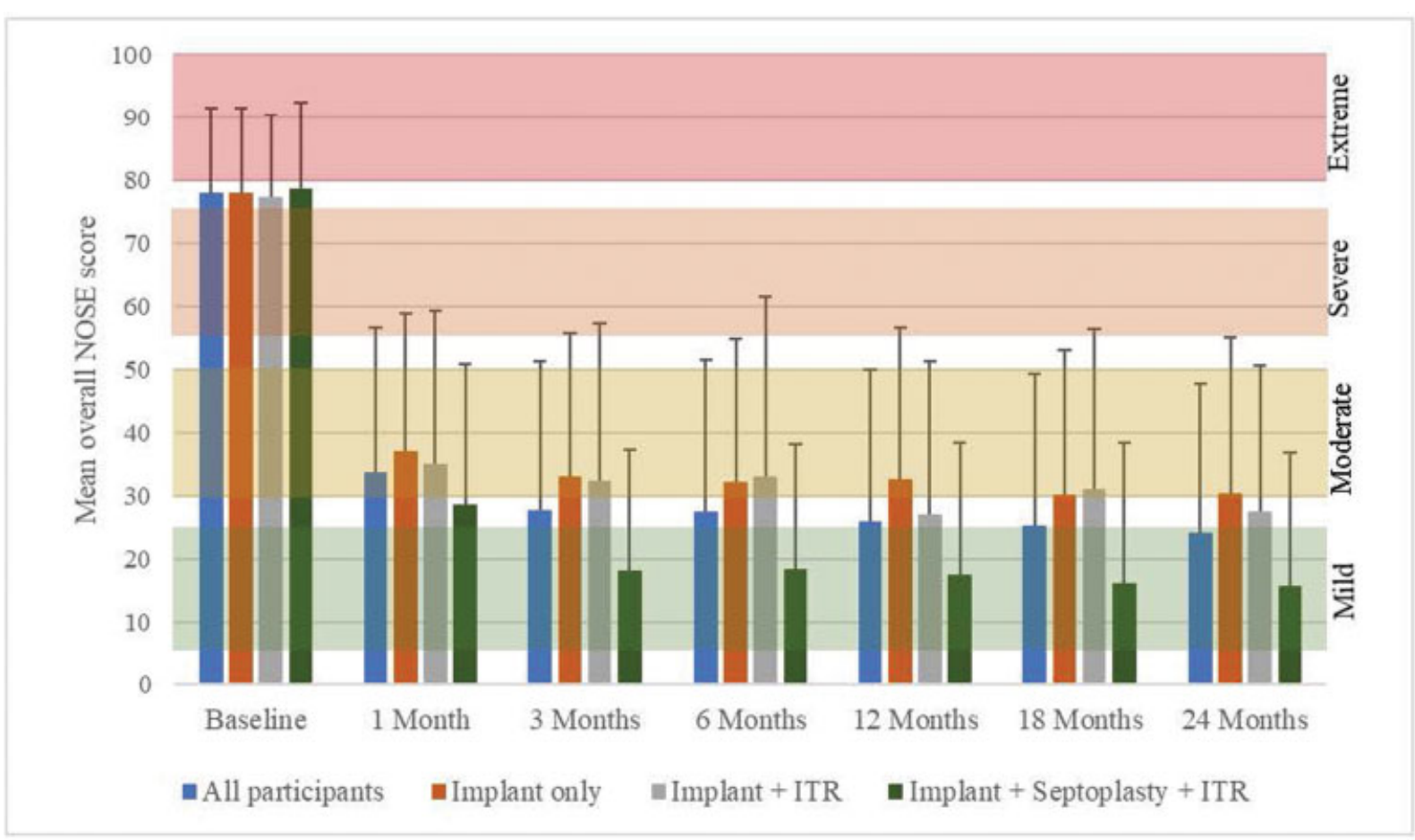

Fig. 1 Change in mean total NOSE Score over time. Nasal Obstruction Symptom Evaluation (NOSE) scores can range from 0 (no symptoms) to 100 (extreme symptoms). Symptom categories were established by Lipan and Most. ${ }^{11}$ Error bars indicate standard deviations. All follow-up visits NOSE scores for each treatment group were statistically improved over baseline $(p<0.001)$. ITR, inferior turbinate reduction.

based on the real-world situation that each patient has different treatment needs and increases the generalizability of the results. While concomitant ITRs (NOSE responder rates $88.1-94.9 \%$ ) did not provide any additional improvement over the implant alone (88.3-94.5\%), for participants who required septoplasty, there appears to be a slight synergistic effect (93.8-95.8\%) to the combined procedure. This suggests the importance of recognizing the contribution of the nasal valve in nasal obstruction. However, the contribution of a revision septoplasty in participants with previously inadequate septoplasty cannot be determined in this cohort. These findings further support the concept of tailoring treatment options to relieve the site of obstruction in each individual and ensuring evaluation of the nasal valve when evaluating patients for nasal obstruction surgery.
Strengths of our study include the prospective multicenter design, the large population, long-term follow-up, and use of validated patient-reported outcomes for nasal obstruction (NOSE and VAS). ${ }^{10,12}$ The large number of participating centers and physicians supports the general adoptability of this procedure in a variety of clinical settings within the United States. By combining studies, we were able to report on the outcomes of 177 participants with 24-month followup. The outcomes at 24 months should be representative of long-term improvement since the implant is absorbed by the body over a period of approximately 18 months. ${ }^{13,14}$

The lack of a control group is the limitation of this study. Although these were single-arm studies comparing pretreatment with post-treatment outcomes within patient, a recent randomized control trial has demonstrated the superiority of the implant over a sham procedure for the treatment of NVC.

Table 4 NOSE responder rates by treatment group

\begin{tabular}{|l|l|l|l|l|l|l|}
\hline Participant group & $\mathbf{1}$ mo & $\mathbf{3}$ mo & 6 mo & 12 mo & 18 mo & 24 mo \\
\hline Implant only & $90.8 \%(99 / 109)$ & $92.5 \%(98 / 106)$ & $92.0 \%(92 / 100)$ & $88.3 \%(83 / 94)$ & $94.5 \%(69 / 73)$ & $89.9 \%(62 / 69)$ \\
\hline Implant + ITR & $88.1 \%(59 / 67)$ & $91.9 \%(57 / 62)$ & $85.7 \%(54 / 63)$ & $90.7 \%(49 / 54)$ & $88.9 \%(40 / 45)$ & $94.9 \%(37 / 39)$ \\
\hline $\begin{array}{l}\text { Implant }+ \\
\text { septoplasty + ITR }\end{array}$ & $93.0 \%(93 / 100)$ & $94.9 \%(94 / 99)$ & $95.8 \%(91 / 95)$ & $95.2 \%(80 / 84)$ & $95.5 \%(64 / 67)$ & $95.7 \%(66 / 69)$ \\
\hline All participants & $90.9 \%$ & $93.3 \%$ & $\begin{array}{l}91.9 \% \\
(237 / 258)\end{array}$ & $\begin{array}{l}91.4 \% \\
(212 / 232)\end{array}$ & $\begin{array}{l}93.5 \% \\
(173 / 185)\end{array}$ & $\begin{array}{l}93.2 \% \\
(165 / 177)\end{array}$ \\
\hline
\end{tabular}

Abbreviations: ITR, inferior turbinate reduction; NOSE, Nasal Obstruction Symptom Evaluation.

Note: A NOSE responder is defined as a participant who has improvement of $\geq 1$ NOSE classes or $\geq 20 \%$ reduction from baseline in NOSE score.

${ }^{a}$ Four participants who had implant plus septoplasty without concomitant ITR are included. 
Table 5 Change in mean VAS scores by treatment group

\begin{tabular}{|c|c|c|c|c|c|}
\hline Follow-up period & $N$ & Baseline VAS score & Follow-up VAS score & $\begin{array}{l}\text { LS mean change in VAS } \\
\text { score }(95 \% \mathrm{Cl})\end{array}$ & $p$-Value \\
\hline \multicolumn{6}{|l|}{ Implant only } \\
\hline $1 \mathrm{mo}$ & 109 & $69.8 \pm 18.1$ & $35.7 \pm 24.5$ & $-34.4(-39.2 ;-29.7)$ & $<0.001$ \\
\hline $3 \mathrm{mo}$ & 106 & $69.8 \pm 18.0$ & $32.2 \pm 24.0$ & $-37.9(-42.8 ;-33.1)$ & $<0.001$ \\
\hline $6 \mathrm{mo}$ & 99 & $69.8 \pm 18.2$ & $33.0 \pm 28.4$ & $-37.2(-42.2 ;-32.2)$ & $<0.001$ \\
\hline $12 \mathrm{mo}$ & 94 & $70.0 \pm 18.0$ & $30.5 \pm 27.0$ & $-39.8(-44.9 ;-34.6)$ & $<0.001$ \\
\hline $18 \mathrm{mo}$ & 73 & $71.3 \pm 16.5$ & $26.2 \pm 25.1$ & $-44.3(-50.2 ;-38.5)$ & $<0.001$ \\
\hline $24 \mathrm{mo}$ & 69 & $71.9 \pm 16.3$ & $26.9 \pm 24.7$ & $-43.8(-49.8 ;-37.8)$ & $<0.001$ \\
\hline \multicolumn{6}{|l|}{ Implant + ITR } \\
\hline $1 \mathrm{mo}$ & 67 & $65.0 \pm 17.5$ & $29.9 \pm 25.5$ & $-34.7(-40.9 ;-28.5)$ & $<0.001$ \\
\hline $3 \mathrm{mo}$ & 62 & $64.6 \pm 17.3$ & $30.9 \pm 28.4$ & $-33.5(-39.9 ;-27.0)$ & $<0.001$ \\
\hline $6 \mathrm{mo}$ & 63 & $64.0 \pm 17.0$ & $26.9 \pm 27.0$ & $-37.3(-43.7 ;-30.9)$ & $<0.001$ \\
\hline $12 \mathrm{mo}$ & 54 & $63.6 \pm 16.4$ & $27.6 \pm 29.2$ & $-36.4(-43.3 ;-29.5)$ & $<0.001$ \\
\hline $18 \mathrm{mo}$ & 45 & $64.0 \pm 17.5$ & $26.1 \pm 26.9$ & $-38.0(-45.6 ;-30.5)$ & $<0.001$ \\
\hline $24 \mathrm{mo}$ & 39 & $64.1 \pm 18.0$ & $24.2 \pm 23.7$ & $-40.0(-48.1 ;-31.9)$ & $<0.001$ \\
\hline \multicolumn{6}{|c|}{ Implant + septoplasty + ITR ${ }^{\mathrm{a}}$} \\
\hline $1 \mathrm{mo}$ & 100 & $64.5 \pm 20.2$ & $23.4 \pm 21.6$ & $-40.4(-44.5 ;-36.2)$ & $<0.001$ \\
\hline $3 \mathrm{mo}$ & 99 & $64.7 \pm 20.4$ & $17.7 \pm 21.1$ & $-46.1(-50.3 ;-42.0)$ & $<0.001$ \\
\hline $6 \mathrm{mo}$ & 94 & $64.4 \pm 20.4$ & $17.6 \pm 21.2$ & $-46.2(-50.4 ;-41.9)$ & $<0.001$ \\
\hline $12 \mathrm{mo}$ & 84 & $62.7 \pm 19.6$ & $15.9 \pm 21.4$ & $-47.4(-51.9 ;-42.9)$ & $<0.001$ \\
\hline $18 \mathrm{mo}$ & 67 & $61.8 \pm 19.4$ & $15.2 \pm 22.6$ & $-47.9(-52.9 ;-42.8)$ & $<0.001$ \\
\hline $24 \mathrm{mo}$ & 69 & $62.1 \pm 19.6$ & $13.5 \pm 21.6$ & $-49.7(-54.7 ;-44.7)$ & $<0.001$ \\
\hline \multicolumn{6}{|l|}{ All participants } \\
\hline $1 \mathrm{mo}$ & 276 & $66.7 \pm 18.8$ & $29.8 \pm 24.2$ & $-36.6(-39.5 ;-33.7)$ & $<0.001$ \\
\hline $3 \mathrm{mo}$ & 267 & $66.7 \pm 18.9$ & $26.5 \pm 25.0$ & $-39.9(-42.9 ;-37.0)$ & $<0.001$ \\
\hline $6 \mathrm{mo}$ & 258 & $66.4 \pm 18.9$ & $25.8 \pm 26.4$ & $-40.6(-43.6 ;-37.6)$ & $<0.001$ \\
\hline $12 \mathrm{mo}$ & 232 & $65.9 \pm 18.5$ & $24.5 \pm 26.4$ & $-41.7(-44.8 ;-38.5)$ & $<0.001$ \\
\hline $18 \mathrm{mo}$ & 185 & $66.1 \pm 18.3$ & $22.2 \pm 25.1$ & $-44.1(-47.6 ;-40.5)$ & $<0.001$ \\
\hline $24 \mathrm{mo}$ & 177 & $66.3 \pm 18.4$ & $21.1 \pm 23.9$ & $-45.3(-48.9 ;-41.7)$ & $<0.001$ \\
\hline
\end{tabular}

Abbreviations: ITR, inferior turbinate reduction; LS, least squares; SD, standard deviation; VAS, visual analog scale (0 to 100 scale). Note: Results are presented as mean \pm SD.

${ }^{\mathrm{a}}$ Four participants who had implant plus septoplasty without concomitant ITR are included.

In the randomized trial, Stolovitzky et al reported a NOSE response rate of $82.5 \%$ at 3 months post implant compared with $54.7 \%$ of a sham procedure $(p<0.001){ }^{15}$ Our treatment response rate of $92.5 \%$ in the implant only group at 3 months is slightly better than those observed in the randomized trial.

A second limitation of our study was that the initial study design only included follow-up through 12 months. The longterm follow-up amendment required additional consent and resulted in some loss to follow-up at long-term visits. Additionally, two patients were withdrawn due to implant retrieval before any follow-up data were obtained. To address this limitation, a worst-case sensitivity analysis was performed. The analysis showed that significant symptom im- provement persisted under these extremely conservative imputation assumptions. Therefore, despite the loss to follow-up, we believe the 24-month results are reliable.

\section{Conclusion}

NVC is commonly overlooked as a factor in nasal airway obstruction. A novel method of addressing the lateral wall deficiency with a bioabsorbable implant is effective; however, since the implant resorbs by 18 months, questions have been raised regarding the durability of the results. This study demonstrates sustained effectiveness at 24 months after treatment, with significant reductions in NOSE and VAS scores, and high responder rates. 
Table 6 Device- or procedure-related adverse events

\begin{tabular}{|l|l|l|}
\hline Adverse event & $\begin{array}{l}\text { Number } \\
\text { of events }\end{array}$ & $\begin{array}{l}\text { Time to } \\
\text { event (days) } \\
\text { Median (IQR) }\end{array}$ \\
\hline $\begin{array}{l}\text { Implant retrieval/ } \\
\text { extrusion/migration }\end{array}$ & 22 & $10.5[21.3]$ \\
\hline $\begin{array}{l}\text { Implant palpable/bumps } \\
\text { on nose }\end{array}$ & 7 & $33.0[150.0]$ \\
\hline Infection & 7 & $13.0[7.0]$ \\
\hline Foreign body sensation & 6 & $92.0[111.5]$ \\
\hline Discomfort/pain & 4 & $118.0[219.0]$ \\
\hline Skin irritation/inflammation & 2 & $46.0[34.0]$ \\
\hline $\begin{array}{l}\text { Mucous production/ } \\
\text { postnasal drip }\end{array}$ & 2 & $2.5[0.5]$ \\
\hline Hematoma & 1 & $1[0]$ \\
\hline Loss of smell/taste & 1 & $2[0]$ \\
\hline $\begin{array}{l}\text { Unintended perforation } \\
\text { of the skin }\end{array}$ & 1 & $25[0]$ \\
\hline $\begin{array}{l}\text { Implant bent or fractured } \\
\text { during placement }\end{array}$ & 1 & $1[0]$ \\
\hline
\end{tabular}

Abbreviation: IQR, interquartile range.

\section{Funding}

This study was supported by Spirox (now part of Stryker).

\section{Conflict of Interest}

D.M.S. reports personal fees (consulting), nonfinancial support (devices), and institutional research support from Stryker Corp. during the conduct of the study. P.S. reports personal fees (consulting), nonfinancial support (devices), and institutional research support from Stryker Corp. during the conduct of the study. E.M.O. reports being employed by Stryker Corp. during the conduct of the study. R.A.O. reports personal fees (consulting), nonfinancial (devices), and institutional research support from Stryker Corp. during the conduct of the study; personal fees from Aerin Medical and from Smith \& Nephew, outside the submitted work. N.E.N. reports nonfinancial support (devices) and institutional research support from Stryker Corp. during the conduct of the study. S.S. reports nonfinancial support (devices) and institutional research support from Stryker Corp. during the conduct of the study.

\section{References}

1 Rhee JS, Book DT, Burzynski M, Smith TL. Quality of life assessment in nasal airway obstruction. Laryngoscope 2003;113(07):1118-1122

2 Clark DW, Del Signore AG, Raithatha R, Senior BA. Nasal airway obstruction: prevalence and anatomic contributors. Ear Nose Throat J 2018;97(06):173-176

3 Chambers KJ, Horstkotte KA, Shanley K, Lindsay RW. Evaluation of improvement in nasal obstruction following nasal valve correction in patients with a history of failed septoplasty. JAMA Facial Plast Surg 2015;17(05):347-350

4 Spirox, Inc. 510(k) Summary - K161191. Created Jun 23, 2016. Accessed November 5, 2020 at: https://www.accessdata.fda.gov/scripts/cdrh/cfdocs/cfpmn/pmn.cfm?ID=K161191

5 Samra S, Steitz JT, Hajnas N, Toriumi DM. Surgical management of nasal valve collapse. Otolaryngol Clin North Am 2018;51(05): 929-944

6 San Nicoló M, Stelter K, Sadick H, Bas M, Berghaus A. Absorbable implant to treat nasal valve collapse. Facial Plast Surg 2017;33 (02):233-240

7 Stolovitzky P, Sidle DM, Ow RA, Nachlas NE, Most SP. A prospective study for treatment of nasal valve collapse due to lateral wall insufficiency: outcomes using a bioabsorbable implant. Laryngoscope 2018;128(11):2483-2489

8 Sidle DM, Stolovitzky P, Ow RA, et al. Twelve-month outcomes of a bioabsorbable implant for in-office treatment of dynamic nasal valve collapse. Laryngoscope 2020;130(05):1132-1137

9 San Nicoló M, Stelter K, Sadick H, Bas M, Berghaus A. A 2-year follow-up study of an absorbable implant to treat nasal valve collapse. Facial Plast Surg 2018;34(05):545-550

10 Stewart MG, Witsell DL, Smith TL, Weaver EM, Yueh B, Hannley MT. Development and validation of the Nasal Obstruction Symptom Evaluation (NOSE) scale. Otolaryngol Head Neck Surg 2004; 130(02):157-163

11 Lipan MJ, Most SP. Development of a severity classification system for subjective nasal obstruction. JAMA Facial Plast Surg 2013;15 (05):358-361

12 Rhee JS, Sullivan CD, Frank DO, Kimbell JS, Garcia GJM. A systematic review of patient-reported nasal obstruction scores: defining normative and symptomatic ranges in surgical patients. JAMA Facial Plast Surg 2014;16(03):219-225

13 Rippy MK, Baron S, Rosenthal M, Senior BA. Evaluation of absorbable PLA nasal implants in an ovine model. Laryngoscope Investig Otolaryngol 2018;3(03):156-161

14 LATERA ${ }^{\circledR}$ absorbable nasal implant system. Instructions for Use . Stryker; Doc\# 4485-001-rB, November 2019

15 Stolovitzky P, Senior B, Ow RA, Mehendale N, Bikhazi N, Sidle DM. Assessment of bioabsorbable implant treatment for nasal valve collapse compared to a sham group: a randomized control trial. Int Forum Allergy Rhinol 2019;9(08):850-856 